Proc. of The Seventh Intl. Conf. On Advances in Applied Science and Environmental Technology - ASET 2017

Copyright $(\odot$ Institute of Research Engineers and Doctors, USA .All rights reserved.

ISBN: 978-1-63248-136-8 doi: 10.15224/ 978-1-63248-136-8-43

\title{
BIODEGRADATION OF POLYPHENOLS BY ARTHOBACTER CITREUS
}

Nichith K R1,Sneha Bhatt2,Mahesh Arvind3

Corresponding author: Department of Chemistry and Biochemistry, Vijaya College, Basavanagudi, Bangalore560004

ABSTRACT: Polychlorophenols such as pentachlorophenols and trichlorophenols are major environmental pollutants. Tetrachlorophenol can be naturally produced while pentachlorophenol is anthropogenic in origin. The main sources of polychlorophenol contamination are from their production, application \& discharge. Polychlorophenols are harmful to all life forms because they disrupt the integrity and function of biological membranes. Their metabolites are also toxic. The most efficient and economical approach for removal of polychlorophenols is bioremediation. Bacteria can degrade polychlorophenols under both aerobic and anaerobic conditions and fungi are able to aerobically metabolize them. The aerobic breakdown of aromatic compounds starts with monooxygenases or dioxygenases that introduce hydroxyl groups into the aromatic rings and further channelizes the metabolites into TCA cycle for the complete mineralization. However polychlorophenols are converted to substituted quinols before ring cleavage. In the present study we have isolated a microorganism capable of degrading high concentrations of polychlorophenols. The microorganism has been identified as Arthrobacter citreus by biochemical studies. Studies have revealed that the enzyme machinery which is capable of degrading ordinary phenols, nitrophenols, halophenols and cresols are also capable of degrading polychlorophenols. Identification of metabolites by TLC and Spectroscopic methods are indicative of the degradative pathway involving the formation of tetrachloroquinone, tetrachloroquinol, trichloroquinol, dichloroquinol, chloromalylacetate and ketoadipate which enters the TCA cycle.

KEYWORDS: Biodegradation, polyphenols, Arthrobacter

INTRODUCTION: Polychlorophenols and their derivatives are widely used as fungicides, pesticides and herbicides. Despite regulation for their usage and release, they remain as the major group of pollutants, highly toxic to humans. Microbial degradation of chemicals in the environment is an important route for removal of pollutants. Bioremediation of pollutants aims to increase the natural rates of biodegradation to produce non toxic end products. Single strain of bacteria is often chosen, so that biochemical interpretations of the degradation process is simplified. Arthrobacter are the basic soil bacteria, but have been found to perform several important functions when the Earth is poisoned by nasty chemicals. Arthrobacter citreus isolated from a contaminated site is known to degrade high concentrations of pencils phenolic derivatives in industrial effluents.

\section{RESULTS AND DISCUSSION:}

Degradation of Trichlorophenols and

Pentachlorophenols occurred in 24

hours. The microorganisms tolerated

Trichlorophenols and Pentachlorophenol

concentrations up to $12 \mathrm{mM}$ and $15 \mathrm{mM}$

respectively. TLC analysis and

spectrophotometric analysis of the

intermediates suggest that

Pentachlorophenol is degraded to

Tetrachloroquinone, then to

Tetrachloroquinol and further to

Trichloroquinol and Dichloroquinol. 
Dichloroquinol is then converted to Chloromalyl acetate and then to maleyl acetate further converted to betaketoadipate which enters TCA cycle.

In case of Trichlorophenols, it is first converted to Dichloroquinol and finally to beta keep adipate similar to Pentachlorophenols which enters the TCA cycle. Pentachlorophenolmonoxygenase, Tetrachloroquinone reductase, Tetrachloroquinol dehalogenase , Dichloroquinol dioxygenase are the key enzymes involved in the above said conversions during the degradation of Pentachlorophenols and Trichlorophenols. The proposed pathway adopted by the Arthrobacter citreus for the degradation of Pentachlorophenols and Trichlorophenols can be confirmed by assaying for the key enzymes.

\section{CONCLUSION:}

The present study reports that the isolated Arthrobacter citreus strain which has been already adopted to grow on phenol has an additional ability to degrade Pentachlorophenols and Trichlorophenols. The study reveals that Arthrobacter citreus strain can effectively degrade Pentachlorophenols and Trichlorophenols even at higher concentrations. The organism is capable of degrading Nitrophenols, Cresols, Polychlorophenols and other toxic aromatic, therefore, it has a high potential for its use in the development of microbial technology for bioremediation. Enzyme studies and understanding the genetics of phenolic degradation will be helpful in designing strategies for bioremediation of contaminated environments. 